\title{
Region-Based Wavelet-Packet Adaptive Algorithm for Sparse Response Identification
}

\author{
Odair A. Noskoski and José C.M. Bermudez \\ Federal University of Santa Catarina \\ Department of Electrical Engineering \\ odair@cefetrs.tche.br, j.bermudez@ieee.org
}

\begin{abstract}
This paper proposes a new wavelet-packet-based algorithm for sparse response identification. The distinctive features of the new algorithm are a region-based strategy for adaptive weight activation and a new deactivation/activation schedule across the transform scales. The new algorithm shows improved performance when compared to existing wavelet-based algorithms with similar characteristics. The new strategies lead to a faster wavelet-packet transform construction and to improved robustness to design parameters when compared to previous solutions. Monte Carlo simulation results show good performances regarding convergence speed and robustness to design parameter choice.
\end{abstract}

Keywords: Adaptive systems, echo cancellation, sparse systems.

\section{Introduction}

Identification of systems with sparse impulse responses is useful in wireless communications, echo cancellation, underwater acoustics and geophysics. Several algorithms exploit sparsity to improve identification efficiency by detecting the significant (active) samples of the unknown response [1, 2, 3, 4, 5, 6,

The solution in 2] is able to identify responses with an unknown number of dispersive regions. It requires a number of preset parameters, which tends to render optimal designs difficult. One of these parameters is the maximum number of active taps. Thus, efficiency is compromised unless a good estimate of this number is available. Ref. 3 incorporates a least-squares (LS) based active tap detection to the NLMS algorithm. Computational complexity is a little above NLMS's if two previously evaluated look-up tables are used. It has been reported that the LS-based detection may fail for impulse responses with large dynamic ranges [1]. Ref. [4] was the first to exploit the wavelet transform (WT) time hierarchy in sparse system identification. The Haar-Basis (HB) algorithm 4 . works from a control scale for which all weights are adapted at every adaptation interval (AI). After each AI, the converged control weights which are larger than a detection threshold activate the weights in the same time hierarchy at the other scales. Then, a new AI begins during which active and control weights are adapted. This approach can be used to identify sparse responses with more than one dispersive region. Because the weight activations are based on a fixed control 
scale, the algorithm may fail for sparse responses which are not rich enough in frequency content [1] or are orthogonal to the control scale basis vectors [4]. Examples can be found among typical network echo path responses [7, 6]. Finally, the necessary adaptation of all the control weights may lead to longer than necessary adaptive filters, increasing computational cost and convergence time. In [5] two short adaptive filters are used. A first filter operates in a partial-Haar transform domain and estimates the location of the peak of the unknown response. The second filter is a short time domain adaptive filter centered about the estimated peak location. Positioning of the second filter uses the time hierarchy of the WT. The approach requires an estimate of the number of active coefficients and is effective for sparse responses with only one dispersive region.

Wavelet packets generalize the wavelet theory to allow flexible frequency domain signal representations [8]. Ref. 6] proposed a wavelet-packet-based (WPB) algorithm for identification of sparse impulse responses with arbitrary frequency spectra. The WPB algorithm uses localization information in both time and frequency. Frequency information is used to adaptively design a discrete wavelet packet transform (DWPT) tailored to the spectral energy distribution of the unknown response. Time information is used to adaptively locate the active weights. WPB was shown to match the results of 4 whenever HB provided good performance. Moreover, WPB provided very good results for responses not suitable for the WT-based approach of [4.

This paper proposes a new wavelet-packet-based algorithm with improved performance when compared to [4,6]. The distinctive features of the new algorithm are based on the following considerations: 1) Most practical sparse responses are composed of short and dense regions of active samples, 2) Wavelet domain representations of typical impulse responses may have different forms for different delays of the effective response [8, Page 147], 3) Long adaptive intervals, which are desirable for accurate active weight estimations, can be combined with frequent weight deactivations to speed-up convergence and reduce complexity. The new algorithm is called Region-Based Wavelet-Packet (RBWP) algorithm.

Unlike HB and WPB, which activate individual weights, RBWP activates regions of weights. This new active region (as opposed to active weight) detection strategy allows for a significant reduction of the required adaptation intervals. RBWP can build the WP transform in about $1 / 2$ of the iterations required by WPB. RBWP uses also a more effective weight deactivation/activation schedule, as compared to WPB. The new schedule allows for a better estimate of the active weights before testing for deactivation. Monte Carlo (MC) simulations show improved convergence and robustness to design parameter choice of RBWP relative to $\mathrm{HB}$ and WPB. RBWP has been successfully tested with all echo responses in [7] using different effective response delays.

The paper is organized as follows. Section 2 briefly reviews the WPB algorithm [6]. Section 3 presents the new region-based activation strategy and weight deactivation/activation schedule that differentiate RBWP from WPB. The computational complexities of RBWP, NLMS, HB and WPB are compared. Section 4 presents MC simulation results and Section 5 concludes the paper. 


\section{WPB Algorithm}

WPB has two phases [6]. In Phase 1 the DWPT is built and a first pass is made in estimating the active weights. Phase 2 refines the detection and estimation of the active weights for the DWPT defined in Phase 1.

\subsection{Phase 1: Construction of the DWPT}

Fig. 1 shows an $M$-level DWPT construction for $M=3 . \tilde{y}(n)$ is the estimate of the desired signal $y(n)$, which is the unknown system output plus an i.i.d. additive noise with power $\sigma^{2} . e(n)$ is the estimation error. Phase 1 starts with a one-level DWPT realized by Haar filters $\boldsymbol{H}_{L}$ (lowpass) and $\boldsymbol{H}_{H}$ (highpass). Input vector $\boldsymbol{x}(n)$ is $N \times 1$. Decimated output vectors $\boldsymbol{z}_{H_{1}}$ and $\boldsymbol{z}_{L_{1}}$ are $N / 2 \times 1$.

Next step is the first AI, $\mathrm{AI}_{1}$. Two $N / 2 \times 1$ adaptive filters with weight vectors $\boldsymbol{w}_{H_{1}}$ and $\boldsymbol{w}_{L_{1}}$ are adapted, where the subscript 1 indexes the DWPT level. The converged weights are then compared to a threshold. Those larger than the threshold are considered to be active weights. This concludes $\mathrm{AI}_{1}$. The second $\mathrm{AI}\left(\mathrm{AI}_{2}\right)$ follows in which only the active weights are adapted. These weights form vectors $\tilde{\boldsymbol{w}}_{H_{1}}$ and $\tilde{\boldsymbol{w}}_{L_{1}}$, which are excited by the pruned transformed signal vectors $\tilde{\boldsymbol{z}}_{H_{1}}$ and $\tilde{\boldsymbol{z}}_{L_{1}}$. With much less weights than in $\mathrm{AI}_{1}, \mathrm{AI}_{2}$ has reduced complexity and convergence time.

Next step of Phase 1 generates the next level of the DWPT. Only the subband with higher energy is again subdivided. Subband energies are estimated by $\left\|\tilde{\boldsymbol{w}}_{H_{1}}\right\|_{2}$ and $\left\|\tilde{\boldsymbol{w}}_{L_{1}}\right\|_{2}$. The weights in the lower energy subband are migrated into a new adaptive vector $\tilde{\boldsymbol{w}}_{a}$, which is excited by signal vector $\tilde{\boldsymbol{z}}_{a}$ with the corresponding signal samples. Weight vector $\tilde{\boldsymbol{w}}_{a}$ will always be adapted from this point on. The higher energy subband is subdivided by a new set of filters $\boldsymbol{H}_{L}$ and $\boldsymbol{H}_{H}$. This leads to transformed signal vectors $\tilde{\boldsymbol{z}}_{H_{2}}$ and $\tilde{\boldsymbol{z}}_{L_{2}}$ at the output of the second DWPT level. The corresponding weight vectors $\tilde{\boldsymbol{w}}_{H_{2}}$ and $\tilde{\boldsymbol{w}}_{L_{2}}$ are initialized by filtering the higher energy subband coefficients $\left(\tilde{\boldsymbol{w}}_{H_{1}}\right.$ or $\left.\tilde{\boldsymbol{w}}_{L_{1}}\right)$ with $\boldsymbol{H}_{L}$ and $\boldsymbol{H}_{H}$. A new AI follows and another decision is made about which subband will be split. The process is repeated for a new DWPT level until the complete DWPT is obtained.

DWPT

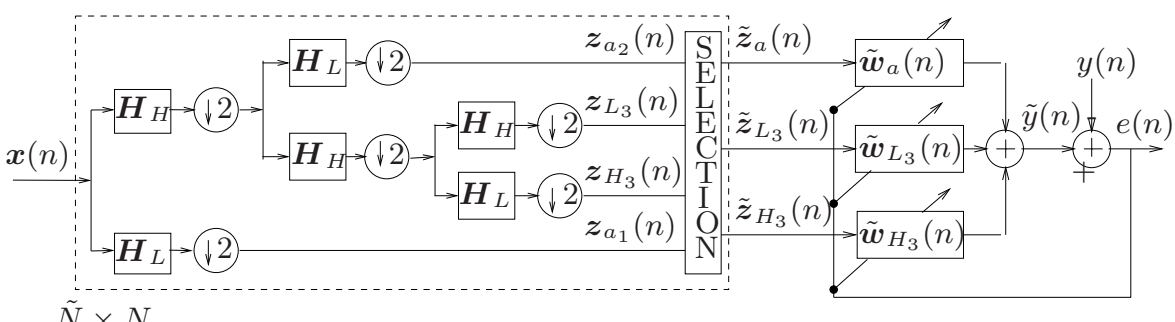

Fig. 1. Block diagram with $M=3$ levels 
Any adaptive algorithm can be used. Here we use the NLMS algorithm 4]. In Fig. 11 $\tilde{y}(n)=\tilde{\boldsymbol{z}}^{T}(n) \tilde{\boldsymbol{w}}(n), \tilde{\boldsymbol{z}}(n)=\left[\tilde{\boldsymbol{z}}_{a}^{T}(n), \tilde{\boldsymbol{z}}_{L_{m}}^{T}(n), \tilde{\boldsymbol{z}}_{H_{m}}^{T}(n)\right]^{T}, \tilde{\boldsymbol{w}}(n)=$ $\left[\tilde{\boldsymbol{w}}_{a}^{T}(n), \tilde{\boldsymbol{w}}_{L_{m}}^{T}(n), \tilde{\boldsymbol{w}}_{H_{m}}^{T}(n)\right]^{T}$ and $e(n)=y(n)-\tilde{y}(n)$. The update equations are: $\tilde{\boldsymbol{w}}_{a}(n+1)=\tilde{\boldsymbol{w}}_{a}(n)+2 \tilde{\mu} \Lambda_{a}^{-2}(n) e(n) \tilde{\boldsymbol{z}}_{a}(n)$ $\tilde{\boldsymbol{w}}_{L_{m}}(n+1)=\tilde{\boldsymbol{w}}_{L_{m}}(n)+2 \tilde{\mu} \lambda_{L_{m}}^{-2}(n) e(n) \tilde{\boldsymbol{z}}_{L_{m}}(n)$ $\tilde{\boldsymbol{w}}_{H_{m}}(n+1)=\tilde{\boldsymbol{w}}_{H_{m}}(n)+2 \tilde{\mu} \lambda_{H_{m}}^{-2}(n) e(n) \tilde{\boldsymbol{z}}_{H_{m}}(n)$

where $\Lambda_{a}^{2}(n)$ is a diagonal matrix of estimates of the power of $\tilde{\boldsymbol{z}}_{a}$ given by $\lambda_{a_{m}}^{2}(n)=(1-\alpha) \lambda_{a_{m}}^{2}(n-1)+\alpha \tilde{z}_{a_{m, 1}}^{2}(n), 0<\alpha<1$, where $m=1,2, \cdots,\left(M^{\prime}+1\right)$ with $M^{\prime}<M$ being the level corresponding to the present adaptive interval. $\tilde{z}_{a_{m, 1}}(n)$ is the first active element of the transformed input vector for level $m$.

\subsection{Phase 2: Adaptation}

The adaptive weights defined by the DWPT constructed in Phase 1 are iteratively adapted and activated/deactivated in Phase 2 according to the temporal hierarchy of the DWPT. Fig. 2 illustrates this hierarchy for $M=5$. The dark rectangles belong to the same temporal hierarchy of element $(3,2)$ (marked with *). Horizontal direction shows distribution in time. Vertical direction shows the 5 levels $(m=1, \ldots, 5)$. Each rectangle corresponds to the region of greater influence of a transformed weight.

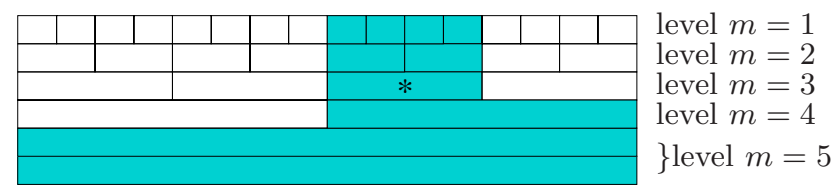

Fig. 2. Hierarchic structure of the DWPT-Haar

WPB processes the input $\boldsymbol{x}$ through the DWPT to determine the reduced transformed vector $\tilde{\boldsymbol{z}}_{a}$ that will excite the adaptive filter with weight vector $\tilde{\boldsymbol{w}}_{a}(n)$, which contains the active weights of all levels. Now, $\tilde{y}(n)=\tilde{\boldsymbol{z}}_{a}^{T}(n) \tilde{\boldsymbol{w}}_{a}(n)$ and $e(n)=y(n)-\tilde{y}(n)$. The weight update equation is

$$
\tilde{\boldsymbol{w}}_{a}(n+1)=\tilde{\boldsymbol{w}}_{a}(n)+2 \tilde{\mu} \Lambda_{a}^{-2}(n) e(n) \tilde{\boldsymbol{z}}_{a}(n) .
$$

\section{RBWP Algorithm}

This section describes the differences between the WPB and the proposed RBWP algorithm.

\subsection{New Region-Based Activation Strategy}

$\mathrm{HB}$ or WPB detect active weights. RBWP detects active regions. After large active weights are detected, neighboring weights about the detected weights are 
aggregated to form active regions. Fig. 3 illustrates the strategy. A sub-region $R_{i}$ at level $m$ is defined about each detected weight. The union $R$ of all $R_{i}$ defines the region of weights to be declared active at level $m$. Weights outside $R$ are deactivated. Weights at level $m-1$ which are in the same time hierarchy of the active region of level $m$ are activated. The spread of each $R_{i}$ about a detected weight is a design parameter. Here we consider one unit to each side, as shown in Fig. 3. This new strategy leads to a significant reduction in the

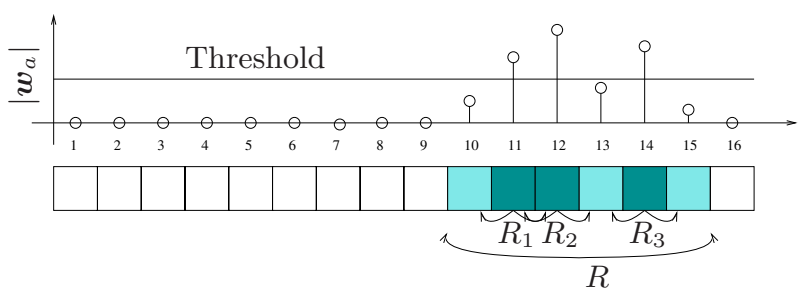

Fig. 3. Example of detection regions for a real response

number of iterations required to build the DWPT in Phase 1. RBWP requires only about 200 iterations for $\mathrm{AI}_{1}$, the most critical step in building the DWPT, for all responses in [7]. This is an order of magnitude faster than WPB. $\mathrm{AI}_{1}$ is then followed by several AIs using the same strategy. After some AIs it is even possible to conclude the DWPT design without new AIs. Only a succession of subband energy estimations and higher energy subband splittings is required, all based on the active samples already identified. The new strategy typically reduces the number of iterations required for WPB to build the DWPT by about 50\%. Thus, the new region-based activation strategy completely modifies Phase 1, as compared to WPB.

\subsection{New Weight Deactivation/Activation Schedule}

Each AI in Phase 2 of RBWP is followed by a region-based weight deactivation/activation step. Besides being region-based, a new schedule is proposed which allows for larger active weight estimation time. Table 1 shows the WPB and the new schedule for $M=5$ and for each cycle of AIs. Take, for instance, level 2 for the RBWP algorithm. The weight activation for this level is done after $\mathrm{AI}_{3}$. Then, the estimation of the weights in this level will be improved over 4 AIs before they are tested for deactivation in $\mathrm{AI}_{2}$ of the next adaptation cycle. In comparison, weights at $m=2$ for the WPB algorithm are activated after $\mathrm{AI}_{4}$ and tested for deactivation right after the next $\mathrm{AI}\left(\mathrm{AI}_{5}\right)$. In $\mathrm{HB}$ [4 weights are also tested for deactivation after each AI. The new schedule clearly allows for either shorter AIs or improved active weight estimations before deactivation tests. This permits a better tradeoff between speed and robustness to design in a given implementation, as compared to HB or WPB. 
Table 1. Deactivation/activation schedule after each AI in one adaptation cycle of WPB and RBWP algorithms for $\mathrm{M}=5$

\begin{tabular}{|c|c|c|}
\hline \multicolumn{2}{|c|}{ Adaptation interval in each cycle } & 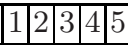 \\
\hline \multirow{2}{*}{ WPB } & Deactivation in level & \begin{tabular}{|l|l|l|l|l|}
1 & 5 & 4 & 3 & 2 \\
\end{tabular} \\
\hline & Activation in level & 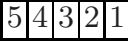 \\
\hline \multirow{2}{*}{ RBWP } & Determination of $R$ in level & \begin{tabular}{|l|l|l|}
1 & 2 \\
\end{tabular} \\
\hline & Activation in & \begin{tabular}{|l|l|l|}
5 & 1 & 2 \\
\end{tabular} \\
\hline
\end{tabular}

\subsection{Computational Complexity}

The exact computational complexity of HB, WPB or RBWP is very difficult to predict. The number of adapted weights varies according to decisions made during identification. Computations required for detections, decisions and step size updatings are implementation dependent. For an $N$-tap response $\left(N=2^{M}\right)$, the number $\tilde{N}$ of weights effectively adapted (NWEA) is, in general, different for each algorithm.

Table 2 shows the number of operations required for a typical iteration of the NLMS 9], HB, WPB and RBWP algorithms as functions of $N$ and $\tilde{N}$. These expressions will be used in the next section for comparison.

Table 2. Computational complexity of the NLMS, HB, WPB, and RBWP algorithms

\begin{tabular}{|c|c|c|c|}
\hline Algorithm & Additions & Multiplications & Divisions \\
\hline \hline NLMS & $2 N+3$ & $2 N+3$ & 1 \\
\hline HB-WPB-RBWP & $2 \tilde{N}+3 M+1$ & $2 \tilde{N}+5 M+4$ & $M+1$ \\
\hline
\end{tabular}

\section{Simulation Results}

This section compares the performances of RBWP, HB and WPB for response $g m_{1}$ in [7] using $N=512$ taps. This is the response from [7] that led to the best results for HB. The detection threshold used for all algorithms was $T H=\beta_{f a} \sqrt{\tilde{\mu} \tilde{\xi}(k) / \tilde{\lambda}_{a_{m}}^{2}(k)}$ [4], where $\tilde{\xi}(k)=(1-\tilde{\mu}) \tilde{\xi}(k-1)+\tilde{\mu} e^{2}(k)$ is an estimate of $\mathrm{E}\left[e^{2}(k)\right] . \tilde{\mu} / \tilde{\lambda}_{a_{m}}^{2}(k)$ is the NLMS step size and $\beta_{f a}$ determines the probability of false alarm [4, Section II-C]. For best performance, $\beta_{f a}=2.56$ for $\mathrm{HB}$ and RBWP, and $\beta_{f a}=0.9$ for WPB. $\boldsymbol{x}(n)$ was generated by passing a white, Gaussian random sequence through the filter $H(z)=$ $0.25 \sqrt{3} /\left(1-1.5 z^{-1}+z^{-2}-0.25 z^{-3}\right)$ to produce a signal with a spectrum closely resembling the spectrum of speech [4, Eq. (29)]. The SNR $\left(\sigma_{x}^{2} / \sigma^{2}\right)$ was $40 \mathrm{~dB}$.

Step sizes were $\tilde{\mu}=1 / 10 \tilde{N}$ for $\mathrm{WPB}$ and $\mathrm{RBWP}$ and $\tilde{\mu}=1 / 8 \tilde{N} \mathrm{HB}$. AIs with $4 \tilde{N}$ iterations were used for $\mathrm{HB}, \mathrm{RBWP}$ and for Phase 2 of WPB. For DWPT building in WPB, $\mathrm{AI}_{1}$ and $\mathrm{AI}_{2}$ of Phase 1 had 2000 and 4000 iterations, respectively. Phase 1 of $\mathrm{RBWP} \mathrm{AI}_{1}$ had 200 iterations. All remaining AIs had 
$4 \tilde{N}$ iterations, up to a total of 3000 , which is $50 \%$ of the number of iterations required for Phase 1 of WPB.

Figs. 4(a) and 4(c) show model $g m_{1}$ of [7] with delays $\delta=253$ and 246, respectively, for a 512-tap channel. Figs. 4(b) and 4(d) show the corresponding WT weights for level 3, the control scale used for HB. Note that the transformed responses are quite distinct [8, Page 147]. Fig. 4(e) clearly shows that the weight at position 33 will be considered inactive for this control scale. The WT time hierarchy will then render weights $129-132$ of level 1 also inactive using the HB algorithm. Fig. 4(f) shows the weights for level 1 of the WT corresponding to Fig. 4(c). It is clear that weights 129-131 should be active. Thus, a poor performance is expected for HB in this case. A similar situation occurs for WPB for $\delta=253$ due to the quasi-orthogonality of $\mathrm{gm}_{1}$ and the DWPT used. Both drawbacks are avoided in RBWP because of the active region detection strategy.

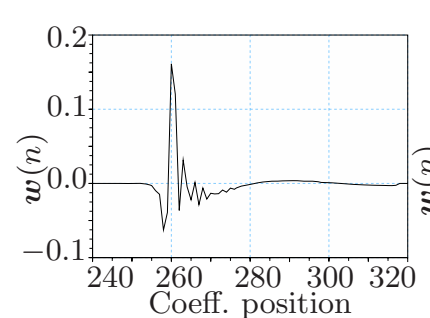

(a)

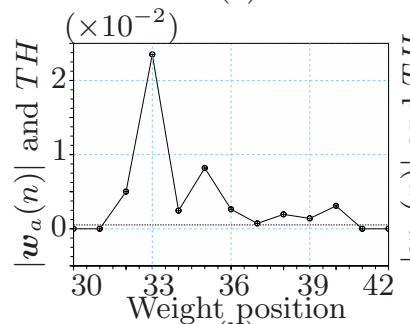

(b)

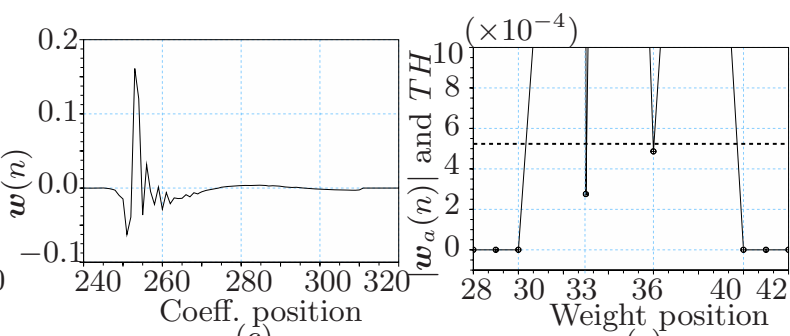

(c)

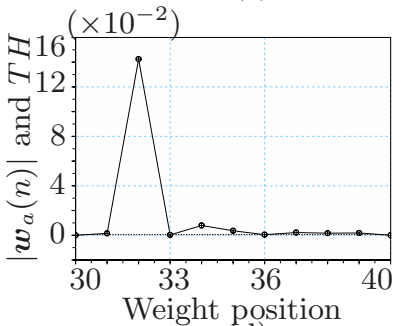

(d)

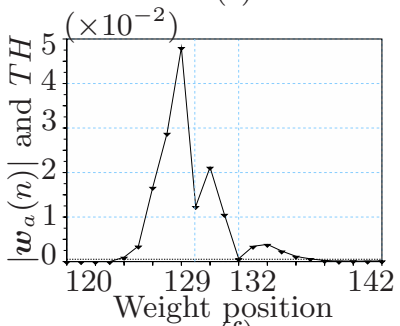

(f)

Fig. 4. (a) Model $g m_{1}$ with delay of $\delta=253$. (b) WT weights for level 3 of signal in (a). (c) Model $g m_{1}$ with delay of $\delta=246$. (d) WT weights for the level 3 of signal in (c). (e) ZOOM of (d). (f) WT weights for level 1 of signal in (c).

Fig. 5 (a) and 5 (b) show MC simulations (100 runs) for the excess mean-square error (EMSE) for $\delta=253$ and $\delta=246$, respectively, for NLMS (time-domain), NLMS-TD (WT-domain), HB, WPB and RBWP algorithms. Note that RBWP presents faster convergence and lower EMSE in both cases. Except for NLMS and NLMS-TD, HB (WPB) had the poorest performance for $\delta=246(\delta=253)$. These results were expected as explained above.

The average NWEA (steady-state) for both delay values were $\tilde{N}=140$ for HB, $\tilde{N}=60$ for WPB, $\tilde{N}=90$ for RBWP. Using Table 2, it is verified that $\mathrm{HB}, \mathrm{WPB}$ and RBWP require about $31 \%, 16 \%$ and $22 \%$, respectively, of the complexity required by time-domain NLMS. 


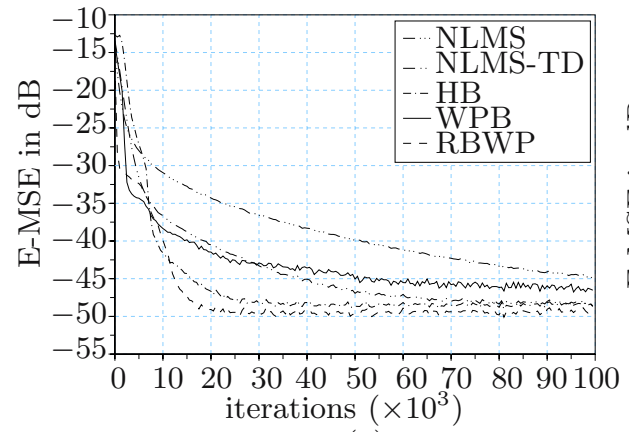

(a)

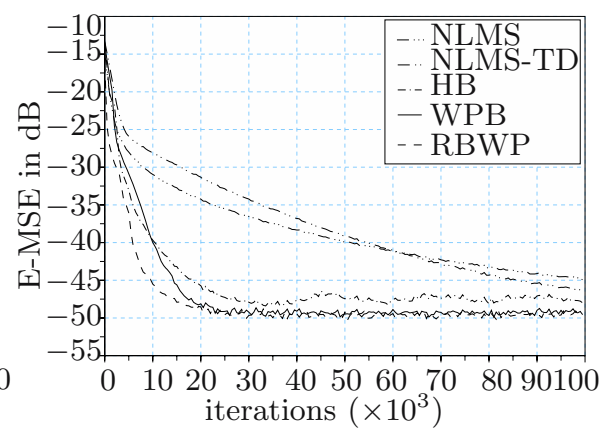

(b)

Fig. 5. Excess mean square error. (a) $\delta=253$. (b) $\delta=246$.

\section{Conclusion}

This paper has presented a new region-based wavelet-packet (RBWP) algorithm for identification of sparse impulse responses with arbitrary spectra. RBWP new region-based activation strategy significantly reduces the time required to build the wavelet-packet transform. MC simulations have shown better performance and robustness to design parameters when compared to existing algorithms.

\section{References}

1. Deng, H., Doroslovački, M.: Proportionate adaptive algorithms for network echo cancellation. IEEE Transactions on Signal Processing 54(5), 1794-1803 (2006)

2. Sugiyama, A., Ikeda, S., Hirano, A.: A fast convergence algorithm for sparse-tap adaptive FIR filters identifying an unknown number of dispersive regions. IEEE Transactions on Signal Processing 50(12), 3008-3017 (2002)

3. Homer, J., Mareels, I.: LS detection guided NLMS estimation of sparse systems. In: Proc. of the ICASSP., vol. 2, pp. 861-864 (2004)

4. Ho, K.C., Blunt, S.D.: Rapid identification of a sparse impulse response using an adaptive algorithm in the Haar domain. IEEE Transactions on Signal Processing 51(3), 628-638 (2003)

5. Bershad, N.J., Bist, A.: Fast coupled adaptation for sparse impulse responses using a partial Haar transform. IEEE Transactions on Signal Processing 53(3), 966-976 (2005)

6. Noskoski, O.A., Bermudez, J.C.M.: Wavelet-packet-based adaptive algorithm for sparse impulse response identification. In: Proc. of the ICASSP., vol. 3, pp. 13211324 (2007)

7. ITU-T Recommendation G. 168: Digital Network Echo Cancellers (2004)

8. Mallat, S.: A wavelet tour of signal processing. Academic Press, London (1998)

9. Sayed, A.H.: Fundamentals of Adaptive Filtering. Wiley, Chichester (2003) 